\title{
IQWiG legt vorläufige Ergebnisse vor
}

Das Institut für Qualität und Wirtschaftlichkeit im Gesundheitswesen (IQWiG) hat am 13. Juli 2015 die vorläufigen Ergebnisse einer Recherche nach evidenzbasierten Leitlinien zur chronischen Herzinsuffizienz vorgelegt.

\section{G-BA diskutiert eigenes DMP Herz- insuffizienz}

Ziel des Berichts ist es, in aktuellen, methodisch hochwertigen Leitlinien diejenigen Empfehlungen zu identifizieren, die für ein Disease-Management-Programm (DMP) von Bedeutung sein könnten. Der Gemeinsame Bundesausschuss (G-BA) zieht in Erwägung, ein eigenes DMP für chronische Herzinsuffizienz einzurichten und beauftragte deshalb das IQWiG mit der Recherche. Bisher gibt es lediglich ein Modul Herzinsuffizienz in- nerhalb des DMP für koronare Herzkrankheit (KHK).

Viele Empfehlungen zu Therapie von Begleiterkrankungen

Breiten Raum nehmen in den Leitlinien - und im Vorbericht - Diagnostik und medikamentöse Therapie ein. Einen weiteren Schwerpunkt bildet die Therapie von Begleiterkrankungen wie etwa KHK, Hypertonie oder Myokarditis. Thematisiert werden aber auch interventionelle Maßnahmen (z.B. kardiale Resynchronisationstherapie, implantierbare Kardioverter-Defibrillatoren), spezielle Personengruppen mit Herzinsuffizienz (Schwangere, Kinder und Jugendliche), die dekompensierte Herzinsuffizienz oder die Palliativversorgung bei terminaler Herzinsuffizienz.

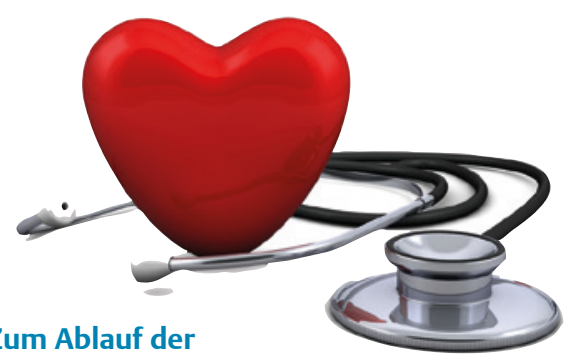

Zum Ablauf der

Berichtserstellung

Den vorläufigen Berichtsplan für dieses Projekt hatte das IQWiG im Januar 2015 vorgelegt und um Stellungnahmen gebeten. Diese wurden zusammen mit einer Würdigung und dem überarbeiteten Berichtsplan im April 2015 publiziert. Stellungnahmen zu dem jetzt veröffentlichten Vorbericht werden nach Ablauf der Frist gesichtet. Sofern sie Fragen offen lassen, werden die Stellungnehmenden zu einer mündlichen Erörterung eingeladen.

Pressemeldung Institut für Qualität und Wirtschaftlichkeit im Gesundheitswesen (IQWiG), 13.07.2015

\section{Typ-1-Diabetes}

\section{Diabetes-Risikotests verhindern Ketoazidose}

Die Ketoazidose ist die führende Todesursache bei Kindern mit Typ-1-Diabetes. Der Diabetes verläuft über Monate manchmal Jahre - unerkannt. Symptome wie übermäßiger Durst, häufiges Wasserlassen oder starke Gewichtsabnahme treten erst auf, wenn schon $80 \%$ der Insulin produzierenden Betazellen in der Bauchspeicheldrüse durch den Angriff der körpereigenen Antikörper zerstört sind und der Körper den Blutzucker nicht mehr regulieren kann. Allerdings lassen sich im Blut schon Jahre vorher Autoantikörper nachweisen. Diese Autoantikörper sind Indikatoren der Autoimmunerkrankung im Blut. Liegen mindestens 2 Autoantikörper vor, entwickeln nahezu 100\% der Betroffenen innerhalb von 20 Jahren einen Typ-1-Diabetes, der durch Insulinzufuhr behandelt werden muss. Dies ergaben Auswertungen des Instituts für Diabetesforschung, Helmholtz Zentrum

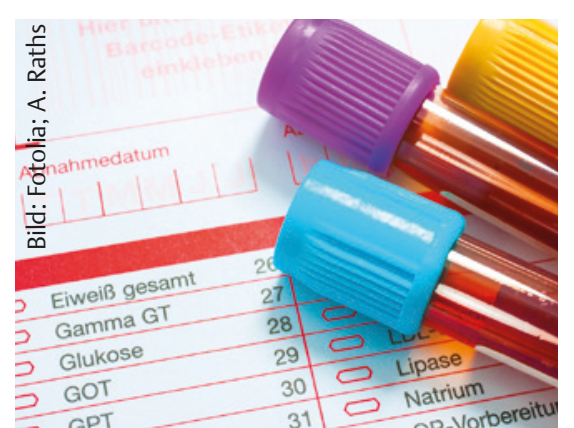

München. Die Forscher sammelten und analysierten weltweit Daten zu 13777 Studienteilnehmern aus Risikofamilien über einen Zeitraum von 20 Jahren.

\section{Milder Verlauf bei früher Diagnose}

„Leider beginnt die Laufbahn eines Typ1-Diabetikers häufig auf der Intensivstation“, so Prof. Anette-Gabriele Ziegler, Direktorin des Instituts für Diabetesforschung und Inhaberin des Lehrstuhls für Diabetes und Gestationsdiabetes der Technischen Universität München. Dies ließe sich mithilfe eines einfachen Risikotests verhindern. Daher hat die praktizierende Diabetologin und Diabetesforscherin das Pilotprojekt Fr1da unter Schirmherrschaft der Bayerischen Staatsministerin für Gesundheit und Pflege, Melanie Huml, initiiert. Bayernweit bieten Pädiater für Kinder im Alter zwischen 2 und 5 Jahren diesen einmaligen Bluttest an. „Die frühe Diagnose soll in erster Linie dazu dienen, lebensbedrohliche Ketoazidosen $\mathrm{zu}$ verhindern und die Familien mit psychologischer Unterstützung und mit Schulungsmaßnahmen auf die Erkrankung und ihre Therapie bestmöglich vorzubereiten“, sagt Ziegler. „Ein Inselautoantikörper-Screening kann das Auf- treten von Ketoazidosen nachweislich verhindern und den Krankenhausaufenthalt bei Ausbruch des Typ-1-Diabetes verkürzen. Außerdem lässt sich ein Diabetes besser behandeln, wenn er frühzeitig diagnostiziert wird“.

\section{Kostenloser Risikotest für Verwandte von Betroffenen}

Junge Verwandte von Typ-1-Diabetikern können ebenfalls von einem Risikotest profitieren: Diesen Risikopersonen bietet das Institut für Diabetesforschung in $\mathrm{Zu}$ sammenarbeit mit dem internationalen Forschungsprogramm TrialNet (Natural History Study, NHS) eine kostenlose Blutuntersuchung an, sofern sie nicht älter als 20 Jahre beziehungsweise bei erstgradiger Verwandtschaft nicht älter als 45 Jahre sind. Ebenso wie bei Fr1da wird Blutserum auf 4 Autoantikörper untersucht, die einen Typ-1-Diabetes ankündigen: Autoantikörper gegen das Insulin (IAA), gegen Glutamat-Decarboxylase (GADA), Tyrosin-Phosphatase (IA2-A) und gegen den Zinktransporter 8 (ZnT8-A). Ein genetischer Test ermittelt außerdem, ob Diabetes-Risikogene vorliegen.

Pressemeldung Forschergruppe Diabetes der Technischen Universität München, 8.7.2015 